\title{
Maestros futuros y TIC
}

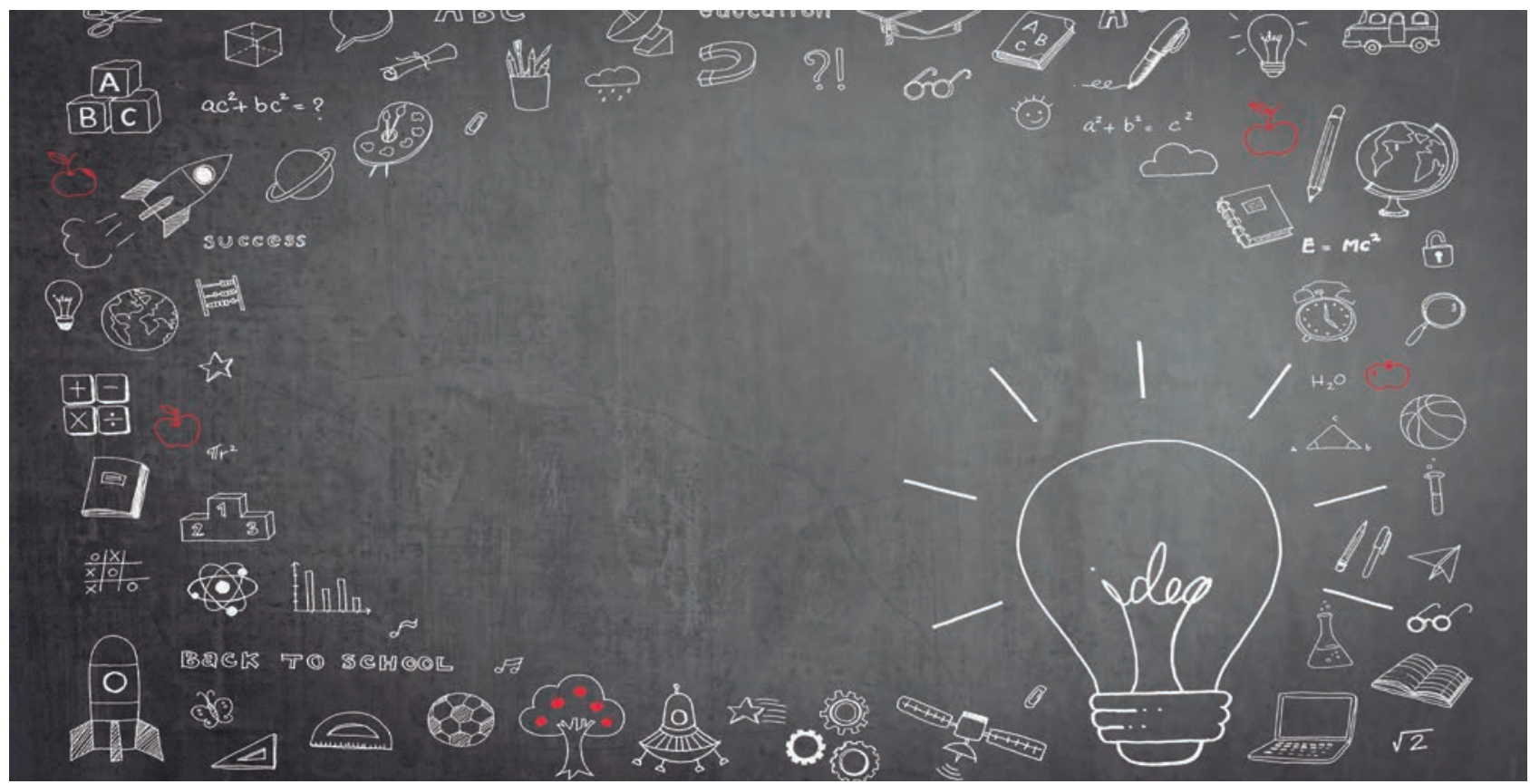

\section{Retos y desafíos de la educación en la cuarta revolución industrial}

La tecnología está cambiando nuestra forma de trabajar, comunicarnos o entretenernos y la educación no puede vivir ajena a esta realidad.

Desde una visión sistémica y con una buena dosis de reflexión y coherencia pedagógica, debemos ser capaces de entender y abordar los retos que ya hoy se nos plantean y los que podemos intuir que están por venir y dotar a nuestros docentes de las competencias digitales y personales necesarias para poder afrontarlos contribuyendo a la mejora de la calidad y equidad del sistema educativo.

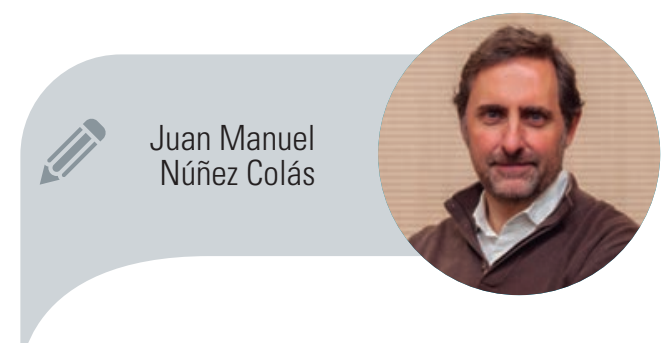

OTBInnova Creative Thinking www.otbinnova.com jnunez@otbinnova.com (Y) @juannunezc

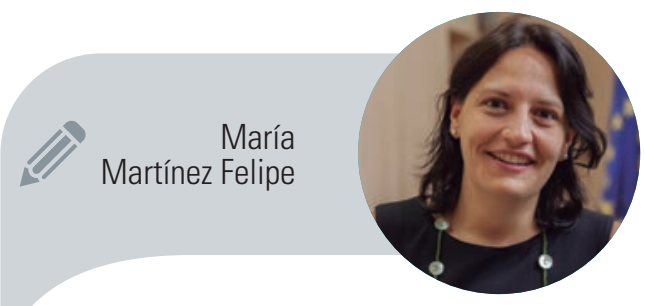

Universidad Pontificia Comillas marfelmar@comillas.edu (y) @marfelmar 

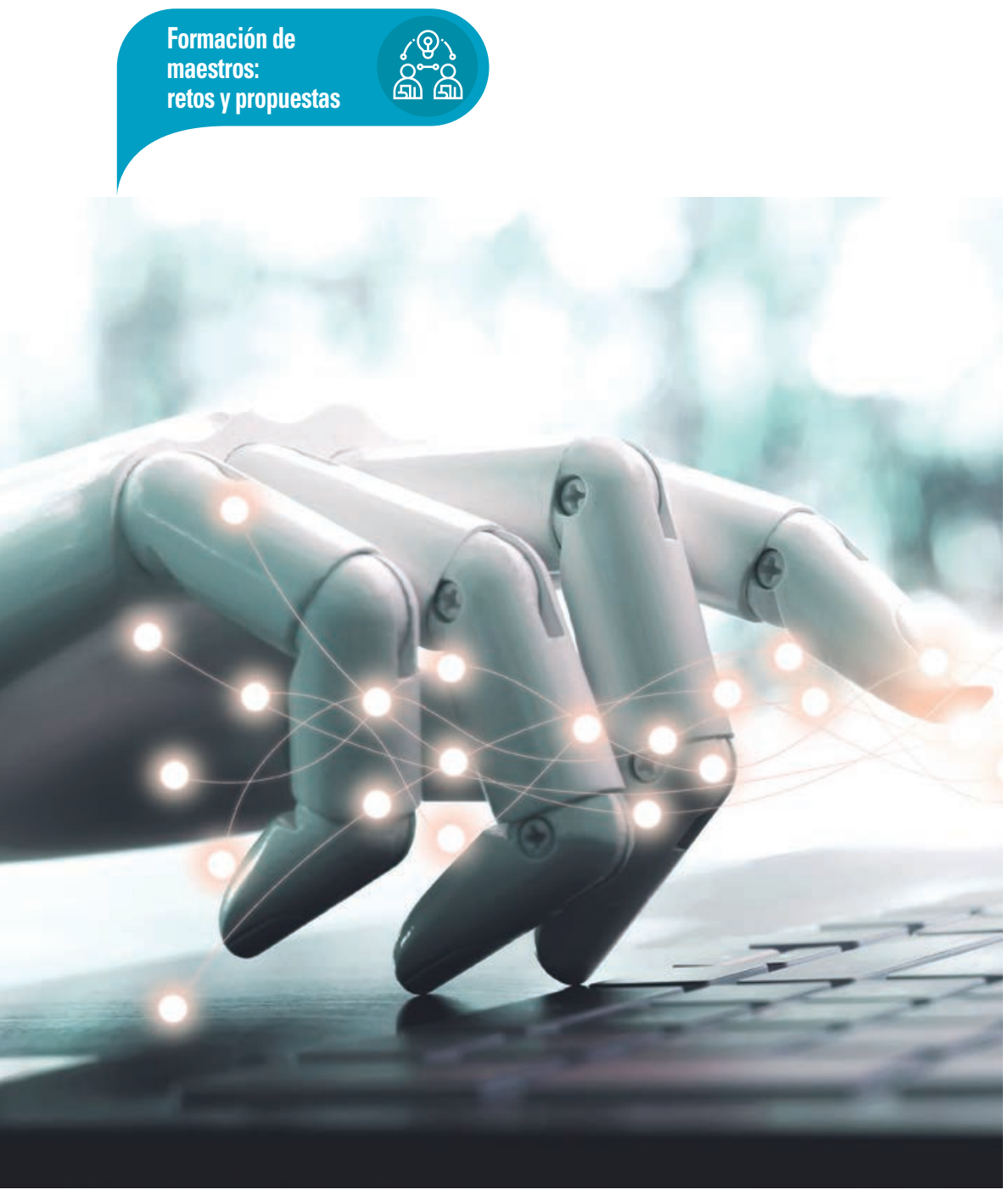

como los actuales que han de prepararse para estos cambios.

Es necesario aproximarse también a estos retos desde una visión sistémica, que nos haga entender que los desafíos de las TIC son múltiples y deben ser observados de forma poliédrica desde todos los ámbitos educativos (procesos, relaciones, pedagogía, espacios, liderazgo, economía, sostenibilidad, etc.).

\section{Retos educativos y TIC hoy}

Los docentes, los equipos directivos y los gestores educativos deben responder cada día a una gran cantidad de retos educativos. Una buena parte de ellos Ilegan de la mano de la tecnología o encontrarán en ella posibles vías de solución. Desde una u otra perspectiva, las TIC jugarán en nuestro día a día educativo un papel crítico de ahora en adelante y cada vez en mayor medida, si bien no debemos olvidar que nuestra tarea es acompañar a los niños y jóvenes en la conformación de su proyecto vital y eso es algo que va mucho más allá de la tecnología y su increíble potencial.

Comenzando en el nivel más general, el sistema educativo debe "tecnificarse" con el objetivo de optimizar procesos y ser capaz de utilizar estos recursos digitales para trabajar por el aumento de la equidad y calidad del sistema en su conjunto. En este nivel identificamos aspectos como la necesidad de tener un modelo de datos único para centros, alumnado y docentes que facilite tanto la movilidad como el análisis de datos, o procesos de gestión documental avanzados que nos ofrezcan buenos repositorios de contenidos o herramientas tanto para la creación como para la docencia y la evaluación, a disposición de todos. Por otro lado, también debe avanzarse en la formación docente online, aunque a este respecto el INTEF (Instituto Nacional de Tecnologías Educativas y de Formación del Profesorado) ha dado pasos interesantes en la buena dirección. Nos guardamos para el final en este punto posibles modelos online de evaluación del profesorado, los centros y los equipos 
directivos, que nos permitan tener certezas sobre la realidad de nuestro sistema, identificar buenas prácticas y promover procesos de mejora basados en evidencias, necesidades e intereses reales.

Cuando nos referimos a los centros educativos, la primera idea a destacar es la necesidad de que cuenten con la autonomía y los recursos necesarios para poder implementar políticas y proyectos TIC que respondan, desde la coherencia pedagógica, a su proyecto educativo y su "perfil de salida del alumno". Desde esta premisa inicial, garantizamos que las TIC estarán al servicio de la educación y no viceversa. A partir de aquí, los retos que plantean las TIC a nivel de centro o agrupaciones de centros es enorme, pero responden en general a dos grandes ejes de trabajo. Por un lado, a todo lo relacionado con la gestión del centro (recursos humanos, espacios, gestión de alumnos, proveedores, etc.) y, por otro, a los procesos de comunicación tanto interna (horizontal y vertical dentro del centro, con los alumnos, las familias) como externa (procesos de marketing, presencia en foros o redes sociales, plataformas o grupos de colaboración presenciales y online, etc.).

Descendiendo en estos niveles de concreción a los que nos estamos refiriendo, nuestros siguientes protagonistas son los equipos directivos que deberían encontrar en las TIC herramientas para optimizar la gestión de proyectos y la generación de procesos de trabajo de equipo y en red a partir de modelos de liderazgo más compartido y transversalizado.

El docente tiene también retos hoy en relación con las TIC, especialmente en relación a la posibilidad de gestionar su aula, planificar, formarse y crear o curar materiales, pero a este aspecto, de especial relevancia, le dedicaremos el próximo punto del artículo.

Y, finalmente, los retos de las TIC en relación con la pedagogía y la didáctica. Aparece aquí la necesaria conversación a tres entre proyecto educativo, metodología y tecnología y a cómo esta puede apoyar aspectos como la evaluación y el aprendizaje basado en evidencias, la atención

\section{ACTIVIDADES DE AULA}

¿Cuál es tu nivel de desarrollo de las competencias digitales docentes? El Instituto Nacional de Tecnologías Educativas y de Formación del Profesorado (INTEF) ha desarrollado un Portfolio de la Competencia Digital Docente que permite tanto el reconocimiento como la mejora de la competencia digital de los docentes. Este servicio tiene en cuenta la autoevaluación continua y el registro actualizado de experiencias de enseñanza, aprendizaje y formación. Enlace: https://portfolio.intef.es/

Plantea una actividad que fomente la innovación educativa:

У Hacer un glosario compartido en la nube con términos de tu materia.

$\checkmark$ Acude a la web de Gamifica tu aula y elige un proyecto.

У Haz que los alumnos monten una infografía o un trivial educativo con la herramienta Genially.

a la diversidad, la inclusión y, en general, todo apoyo que las TIC puedan hacer a la innovación metodológica y educativa en el día a día de los procesos de enseñanza y aprendizaje. Los creadores de material educativo tienen aquí mucho que decir y los necesarios avances en la interoperabilidad de datos y contenidos ayudará sin duda a socializar cualquier avance.

Después de este rápido repaso a los retos a los que se enfrentan los distintos agentes educativos en relación a las TIC, centremos nuestra mirada en las competencias docentes y el estado de la cuestión a este respecto.

\section{Competencias docentes}

La UNESCO, junto con otras instituciones como UNICEF, el Banco Mundial, el UNFPA, el PNUD, ONU Mujeres y el ACNUR organizó el Foro Mundial sobre la Educación 2015 en Incheon, en la República de Corea. Tras dicho foro se publicó la denominada Declaración de Incheon para la Educación 2030 en la que se presentó una visión de la educación para los próximos 15 años. Dentro del preámbulo 10, se fijaba un compromiso para la promoción de oportunidades de aprendizaje de calidad para todos a lo largo de la vida, en todos los contextos y niveles educativos, así como un compromiso para fortalecer la ciencia, la tecnología y la innovación. Esta declaración insistía en servirse de las TIC para reforzar los sistemas educativos, difundir conocimientos, acceder a la información, mejorar la eficacia de los servicios y potenciar un aprendizaje efectivo y de calidad. 
Figura 1. Visión general del marco DigCompEdu

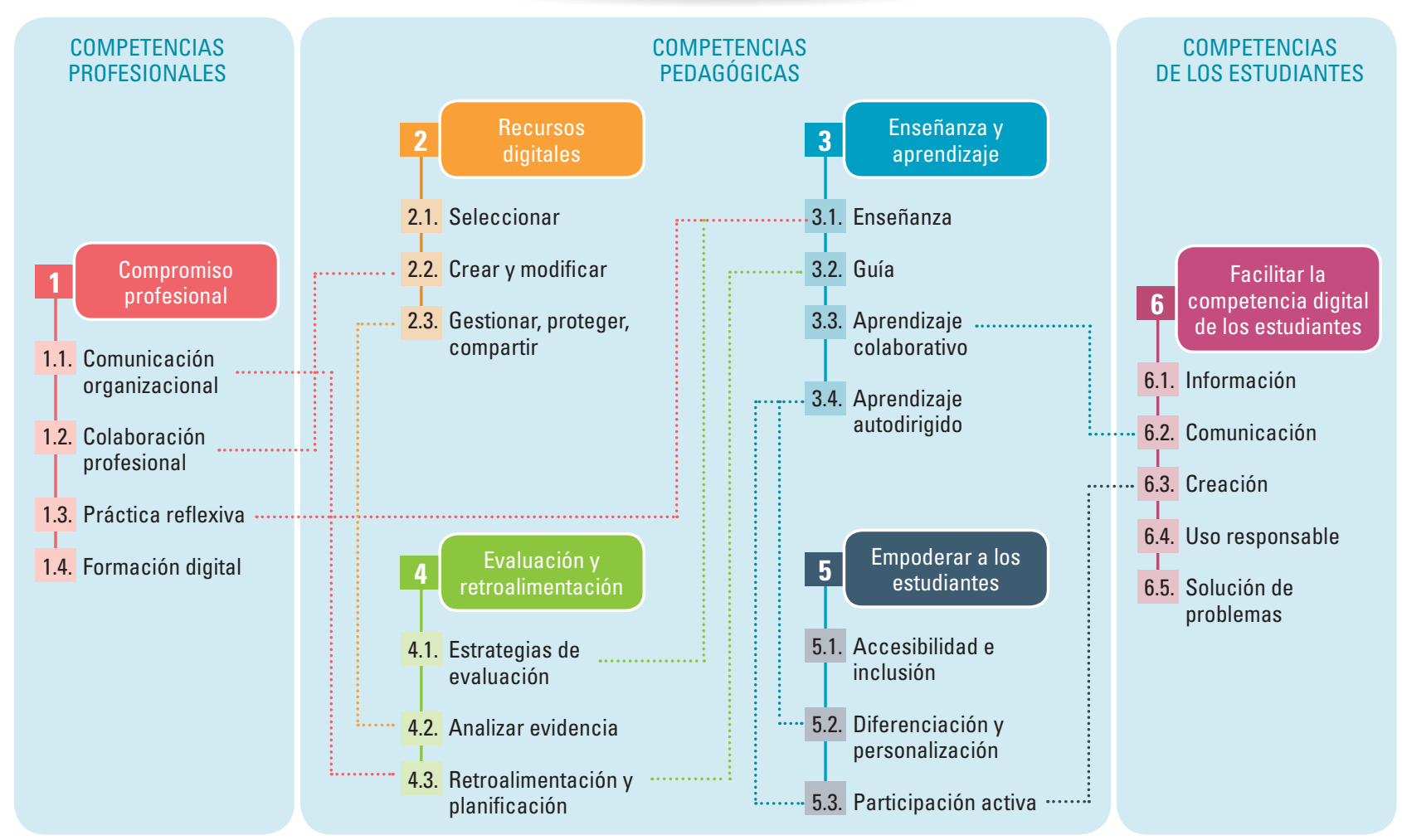

Fuente: https://ec.europa.eu/jrc/digcompedu

Desde hace años la UNESCO ha dado un especial protagonismo al desarroIlo de las competencias digitales al ser esenciales para el empleo y la inclusión social. Recientemente, en 2019, publicó la tercera versión del Marco de competencias de los docentes en materia de TIC UNESCO, diez años después de la publicación del documento original. En esta última versión, se pretende responder a la inminente evolución tecnológica y pedagógica incorporando principios inclusivos de no discriminación, acceso abierto y equitativo a la información e igualdad de género, y donde las TIC desempeñan un papel clave. También se abordan las repercusiones de los recientes avances tecnológicos en materia educativa, como la inteligencia artificial (IA), las tecnologías móviles, la internet de las cosas y los recursos educativos abiertos, en apoyo a la creación de sociedades del conocimiento inclusivas.

La Comisión Europea, también desarrolla un marco europeo para la competencia digital docente, más conocido como DigCompEdu, que se estructura en 6 áreas: 1) compromiso profesional; 2) fuentes, creación y distribución de recursos digitales; 3) cómo armonizar el uso de herramientas digitales en la enseñanza y el aprendizaje (pedagogía digital); 4) evaluación y retroalimentación; 5) empoderamiento a los estudiantes y, por último, 6) cómo facilitar la competencia digital de los estudiantes. Tal y como se puede observar en la figura 1, el área 1 atiende a las competencias profesionales del docente; las áreas 2 a 5 integran el núcleo pedagógico del marco detallando las competencias que los educadores deben desarrollar para fomentar estrategias de aprendizaje efectivas, inclusivas e innovadoras, utilizando herramientas digitales; $y$, por último, el área 6 se centra en las competencias digitales de los estudiantes.

Este marco se dirige a los docentes de todos los niveles educativos, desde infantil hasta la educación superior y de adultos, incluida la formación general y profesional, la educación para las necesidades especiales y los contextos de aprendizaje no formal.

A la hora de evaluar el desarrollo de dichas competencias, se establece un nivel progresivo que se inicia en el nivel A1 
(novel) hasta un nivel máximo de C2 (pionero), al igual que ya ocurría con el Marco Común Europeo de Referencia para las Lenguas.

Los noveles (A1) son aquellos que no han tenido mucha experiencia con herramientas digitales y necesitan orientación para ampliar su repertorio. Los exploradores (A2) empiezan a utilizar herramientas digitales, aunque sin seguir estrategias comprensivas o consistentes aún. Necesitan inspiración para ampliar sus competencias. Los integradores (B1) utilizan herramientas digitales para una variedad de propósitos entendiendo qué estrategias digitales funcionan mejor en función del contexto. Los expertos (B2) experimentan una variedad de herramientas digitales con confianza, de manera creativa y crítica, con el fin de mejorar sus prácticas. Los líderes (C1) disponen de un amplio repertorio de estrategias digitales eficaces, flexibles y completas y además sirven de inspiración para otros. Los pioneros (C2) lideran la innovación, siendo un modelo a seguir para otros profesores y se cuestionan las prácticas digitales y pedagógicas contemporáneas.

Si descendemos del nivel supranacional (UNESCO), pasando por el europeo (DigCompEdu), nos queda por mencionar el Marco común de competencia digital docente, desarrollado por el ya mencionado INTEF en nuestro país. Su finalidad es tanto el diagnóstico como la mejora en sí de aquellas competencias que necesitan desarrollar los docentes del siglo XXI para la mejora de su práctica educativa y para el desarrollo profesional continuo. Al igual que ocurre con el marco europeo ya mencionado, se establece un nivel progresivo desde el A1 (nivel básico) hasta el C2 (nivel avanzado). En esta ocasión hay cinco áreas que configuran la competencia digital docente: 1) información y alfabetización informacional; 2) comunicación y colaboración; 3) creación de contenidos digitales; 4) seguridad; y, por último, 5) resolución de problemas.

Para promover la adquisición, el desarrollo y la mejora de la competencia digital de los docentes, el INTEF ha de-
Figura 2. Progresión del desarrollo de la competencia digital según el marco DigCompEdu

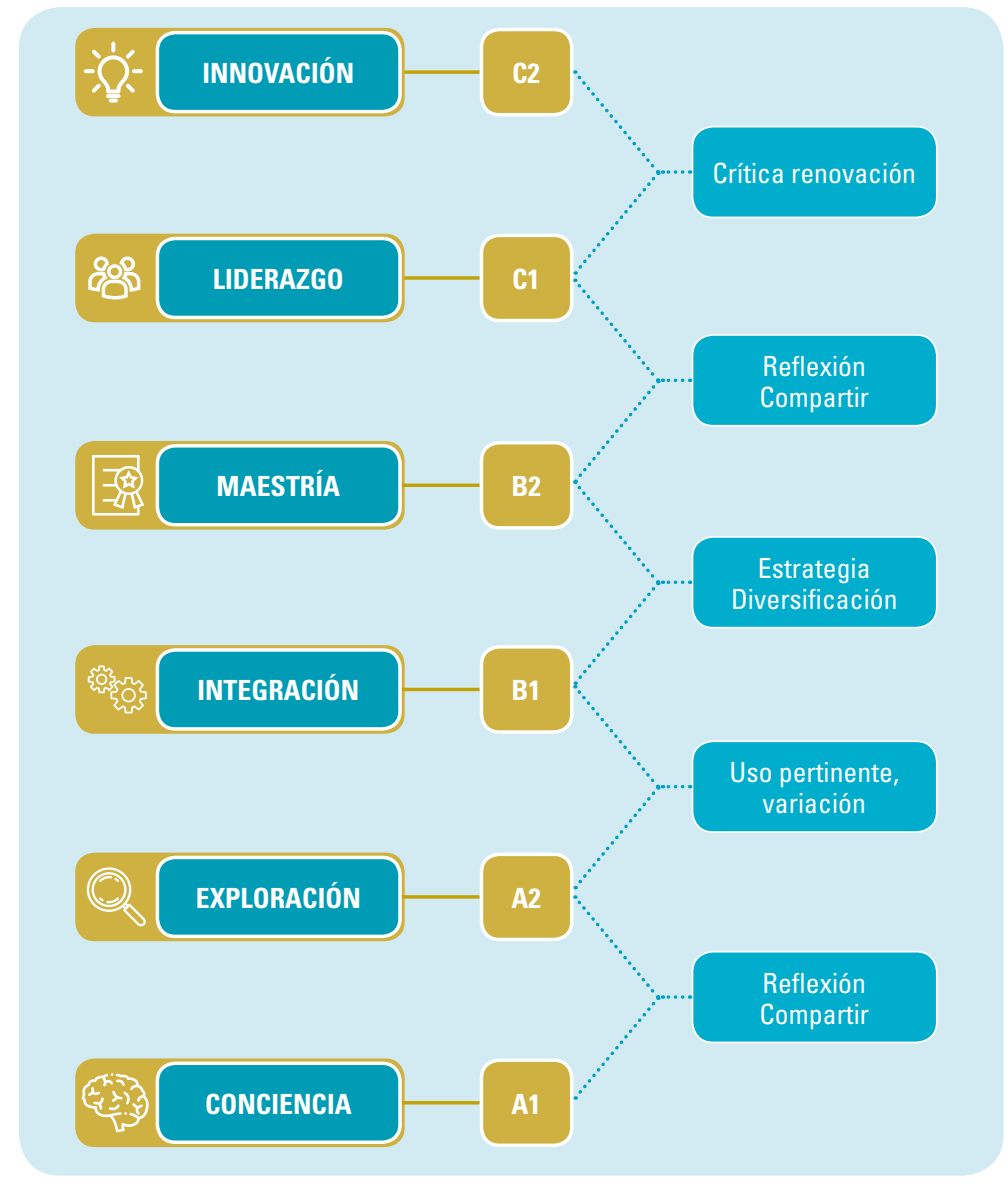

Fuente: https://ec.europa.eu/jrc/digcompedu

sarrollado un Portfolio de la Competencia Digital Docente que se basa en una biografía de datos personales y profesionales, una autoevaluación acorde con las cinco áreas propuestas, un porta-evidencias que pueda avalar su competencia digital docente (insignias, certificados, proyectos, trabajos con alumnos y en centros educativos, premios o publicaciones, entre otros) y, por último, un pasaporte que refleje el nivel alcanzado en dicha competencia, así como una visión general de las evidencias que lo avalan.

\section{Retos educativos y TIC a futuro (no lejano)}

Una vez que tenemos la foto de los actuales retos de las TIC en relación con la educación y de cómo se plantea a nivel nacional e internacional la capacitación y competencia docente para hacer frente a estos desafíos, veamos también dónde 


\section{IIII. ÁQORA DE DROFESORES}

Aprendizaje en línea para una transformación digital de la educación: si quieres estar actualizado, formarte, compartir tus experiencias o saber qué hacen otros profesores como tú... no dudes en acudir al espacio de AprendeINTEF. Allí encontrarás un blog de artículos y noticias (AprenDiario), pequeñas píldoras educativas organizadas por temas (EduPills), sesiones de formación en directo, podrás aprender y compartir experiencias de aprendizaje 0 conocer otros proyectos similares (TeachUp o Act Project, entre otros), Enlace: http://aprende.intef.es/

\section{caminanoo juntos}

Se puede plantear, por ejemplo, un taller relacionado con el aprendizaje-servicio sobre temas básicos de informática y tecnología para abuelos que sean impartidos por alumnos. el fomento del pensamiento computacional y por otro como el trabajo con robots y en espacios maker, que están llamados a convertirse en los grandes espacios de trabajo por proyectos, de empoderamiento, de trabajo en equipo y de fomento de la creatividad de nuestros alumnos y alumnas.

La relación pedagogía y TIC a futuro tiene su gran desafío en la personalización del aprendizaje que debería llegar de la mano de la innovación educativa, del contenido digital adaptativo y de la explotación pedagógica de los desafíos expresados en el párrafo anterior. La tecnología debe ser capaz, a medio plazo, de permitirnos explotar todos los datos que el sistema en su conjunto sea capaz de generar para ofrecer experiencias de aprendizaje adaptadas y activas.

En conclusión, debemos tomar conciencia de la complejidad que supone la digitalización de nuestro sistema educativo y nuestros centros y de lo estratégico de abordarla con la urgencia de los avances tecnológicos continuos y la calma de la reflexión pedagógica y entendiendo que, para hacerlo bien, será necesaria la participación de todos los agentes educativos y una apuesta política decidida en este ámbito con planes ambiciosos desde las administraciones públicas, el sector privado y las instituciones y organizaciones educativas

\section{DARA SABER MÂS}

GHomi, M., y ReDeCKer, C. (2018). Digital Competence of Educators (DigCompEdu): Development and Evaluation of a Self-Assessment Instrument for Teachers' Digital Competence. Berlin: Joint Research Center.

UNESCO. (2015). Declaración de Incheon y marco de acción para la realización del objetivo de desarrollo sostenible 4. París: UNESCO. Recuperado de https://unesdoc.unesco.org/ark:/48223/pf0000245656_spa_

UNESCO (2019). Marco de competencias de los docentes en materia de TIC UNESCO. Tercera Versión. París: UNESCO. Recuperado de https://unesdoc.unesco.org/ark:/48223/pf0000371024?posInSe $\mathrm{t}=2$ \&queryld =53a48924-29bd-49ad-8029-aeeab0c725b4

\section{(-) HemOS HABLADO DE \\ Tecnología de la información; tecnología educacional; informática educativa; habilidad pedagógica; competencias del docente; formación de docentes.}

Este artículo fue solicitado por PADRES Y MAESTROS en septiembre de 2019, revisado y aceptado en febrero de 2020. 